\title{
DETC2009/DAC-87534
}

\section{USAGE COVERAGE MODEL FOR CHOICE MODELING: PRINCIPLES}

\author{
Bernard Yannou, Jiliang Wang, Ndrianarilala \\ Rianantsoa \\ Ecole Centrale Paris \\ Laboratoire Genie Industriel \\ Grande Voie des Vignes \\ 92290, Chatenay-Malabry, France \\ bernard.yannou@ecp.fr \\ Fabrice Alizon \\ Keyplatform, Managing Director \\ 91, rue du Faubourg Saint Honoré \\ 75008, Paris, France \\ fabrice.alizon@keyplatform.com
}

\author{
Chris Hoyle, Mark Drayer, Wei Chen \\ Mechanical Engineering \\ Northwestern University \\ 2145 Sheridan Road, Tech B224 \\ Evanston, IL 60208-3111 \\ weichen@northwestern.edu
}

\author{
Jean-Pierre Mathieu \\ AUDENCIA Nantes Business School \\ 8 , route de la Joneliere, BP 31222 \\ 44312 Nantes Cédex 3, France \\ jpmathieu@audencia.com
}

\begin{abstract}
Accurately capturing the future demand for a given product is a hard task in today's new product development initiatives. As customers become more market-savvy and markets continue fragment, current demand models could greatly benefit from exploiting the rich contextual information that exists in customers' product usage. As such, we propose a Usage Coverage Model (UCM) as a more thorough means to quantify and capture customer demand by utilizing factors of usage context in order to inform an integrated engineering design and choice modeling approach. We start by presenting the principles of the UCM model: terms, definitions, variable classes and relation classes so as to obtain a common usage language. The usage model exhibits the ability to differentiate between individuals' product performance experiences. With Discrete Choice Analysis, individuals' performance with a given product is compared against that of competitive products, capturing individual customers' choice behavior and thereby creating an effective model of product demand. As a demonstration of our methods, we apply our model in a case study regarding the general task of cutting a wood board with a jigsaw tool. We conclude by presenting the scope of future work for the case study and the contribution of the entire current and future work to the field as a whole.
\end{abstract}

\section{INTRODUCTION}

Customer demand models are becoming more prevalent in research works involving the design of products or product families. Accurately capturing customer demand is essential because it can allow for the prediction of future demand for a given product within given market segmentations. The utility of these models is dependent upon the inclusion of an accurate model of customer choice. In the marketing domain, choice models typically consider that marketing attributes, such as brand effect, marketing claims, etc., dominate the choice process [1]. Additionally, customer segmentations have traditionally been based on correlations between these marketing attributes and demographic attributes of the customers. Marketing attributes have generally been a mixture of functional characteristics or product-service performances, design characteristics, perceived quality indicators, marketing claims, purchasing price, and other related services and logistics. Demographic attributes usually account for socioeconomic attributes of the consumers, e.g. the gender, age, where the customer lives, income, occupation, and frequently purchased products in similar or identical product categories.

Under many circumstances, however, these traditional marketing models are not as effective as they could be in driving an efficient marketing policy. For instance, this can occur when the customer is experienced and able to imagine the 
probable performances of the product or service in regard to his or her future usage. In this case, if the customer is well educated about available offerings and able to virtually appreciate and mentally test a proposed product or service, he or she may not be influenced by subjective assessments, brand effect, and marketing claims, limiting the effectiveness of current modeling practices. The utility of a marketing model can also falter when there is a strong presence of competition in a market. Heavy product competition can fragment markets into multiple technical niches, becoming difficult to capture with traditional modeling attributes. Similarly, traditional methods lose effectiveness if disruptive innovation has recently appeared in the market. As soon as the landscape of technological innovations is changing rapidly, past customer behavior is no longer reliable to predict forthcoming product share. In such cases, it may be necessary to utilize analysis tools to investigate the reasons behind and the circumstances under which a given product is being used. Consequently, a more proactive prediction model is required, such as one that consists of studying the actual product usages of the customers, as well as the degree and extent of usages covered by a given productservice offer.

In this way, we see that we can no longer content ourselves with traditional models of customer behavior; rather, we must delve into a study of the reasons behind such behavior. Studying real product usages, with configurations, contexts, and particularities requires design methods and competencies. As a result, the potential of choice modeling in engineering design has not been fully realized due to an overreliance on marketing and demographic attributes to approximate the complex drivers behind customer choice. Customers are growing increasingly technology-savvy and market-educated as more websites offer product comparisons and on-line testimonies. The circumstances outlined above are quickly becoming the rule instead of the exception. Therefore, as the nature of the customer changes and markets fragment, our paradigms must evolve to more effectively and accurately capture the many nuances of customer demand. The prior models built upon the tradition of segmenting markets by product type, price, and customer demographics do not offer insight into why choices were made. Instead, these old models use the measured effects of customer demand in an attempt to recreate the causes because they capture the causes in an incomplete way. Understanding and modeling these causes the reasons behind the choice of a product - requires a new way of thinking about the customer-product relationship.

The marketing field has long been engaged in studying and conceptualizing the driving factors behind customer demand. Ratneshwar and Shocker [2] found that customer product choices are made in the context of specific situational goals and, therefore, are likely to be based on benefits directly related to achieving such goals. Results also showed that products which would otherwise not be considered comparable could and would often be used in the same usage context. Current methods of modeling do not take into account any such situational goals, despite the heavy influence that they have in the customer decision-making process. Additionally, segmenting the market through product type could prove to be detrimental to model effectiveness in cases where nominally diverse products share the same usage context.

In more recent marketing literature, Christensen, Cook, and Hall [3] recommend eliminating the common practice of segmenting markets solely based upon product type, price, and demographics. Instead, they advise to reconsider the underlying concepts that drive customer choice. The proposed new paradigm is that customers seek out products because they have jobs that need to be done, and they hire products to do those jobs for them. The customer is viewed as an "employer of products," one who simply purchases the product that can best complete the task, while the product is viewed as a "provider of services," which is more or less able to meet a need. These "jobs" are inherently rife with contextual factors and in this way, each job is essentially a unique usage context. Current methods of modeling attempt to predict customer choice through demographic attributes, and by doing so, ignore the rich contextual information that can be gained by viewing the customer as a product employer and the product as a service provider. Therefore, in order to model customers as more than irrational, random decision makers, we adopt the assumption that the customer is both weakly influenced by subjective assessments, and that he or she exhibits a rational attitude when matching a product to expected usage contexts. In a second stage, further subjective attributes will be integrated into our model, as we have done in the past in Petiot and Yannou [4].

While the formal study of usage context permeated the field of marketing years ago, it has only just begun to be applied directly to the design and engineering of new products. Green et al. have published three successive papers on the subject [5-7], with the goal of forming a comprehensive product design methodology that includes contextual factors. Important first steps in the field were taken, including the definition of key terms and concepts. Usage context, as it relates specifically to products, is defined as the unique combination of application and environment in which a product is used. Furthermore, usage context is framed as one part of a larger product design context, which also includes market and customer context. This hints at the key role that all three contexts play in guiding the choice of the customer. During the course of the studies, customers were found to have distinct product preferences under different usage contexts. Additionally, evidence supported that contexts could be differentiated based upon functional attributes, indicating a link between engineering parameters and perceived usefulness, which occurs under the influence of different usage contexts. This underscores the benefits of going far beyond using rough functional attributes, as done in Coatanea et al [8].

Choice modeling provides a parametric model to describe how consumers trade-off among multiple design attributes when selecting a product. While the use of choice modeling in 
the context of product design has been investigated recently, most existing choice modeling approaches in design only consider simple design artifacts (e.g., cordless power tools $[9,10]$, bathroom scales $[11,12]$, electric motors $[13,14]$ in which the quantification of product performance is straightforward (e.g., horsepower, weight, power consumption). The effect of usage context and its influence on product performance in choice modeling has not been explicitly considered in the design literature. In our previous work [15], a set of demographic descriptors was presented, which included usage context as a demographic descriptor, as shown in figure 1 ; however, the use of usage context in choice modeling was not developed.

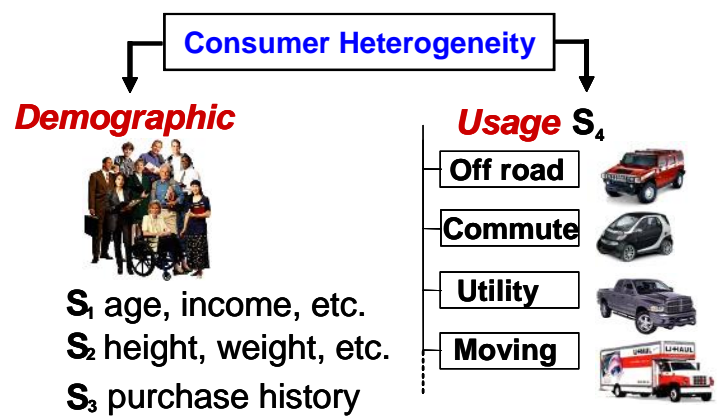

Figure 1: Set of customer descriptors in [15]

The common thread that runs through the literature is the idea that a specific usage context exists in all instances of consumer demand and has a great influence on consumers' satisfaction with a product, and therefore, consumer choice. By ignoring these factors and basing segmentation and customer choice on demographics, the current models are underperforming - they are failing to utilize one of the greatest driving forces behind customer demand.

We propose a new, more comprehensive and more refined demand model that utilizes usage context factors to inform an integrated engineering design and choice modeling approach. The key aspect of our approach lies in our model's ability to account for differences in product performance between different individuals, which previous models have been incapable of achieving. This is accomplished by first considering an individual's usage context as a function of his or her specific usage environment and personal skill level. The quantified usage contexts are then integrated with products' engineering design parameters in a physics-based model to provide a performance prediction for each usage context. Limits are imposed on this generated performance space in order to reduce the amount of survey data collection and choice model computations. This also ensures that the resultant choice set will represent feasible choices in which a customer can make trade-offs among useful attribute values. The limits take the form of a set of acceptable bounds, which restricts the performance space to those performances that would be deemed permissible by the customer. Additionally, the customer may also have a set of preferred bounds which are not only acceptable, but also preferred to other given performances. In order to understand how customers make tradeoffs among these individual performance levels, the bounded performance space is integrated with traditional demographic attributes into a utility function that forms the basis of the choice model. These demographic attributes attempt to capture factors that influence customer product preference. Finally, the augmented customer choice model is integrated into the choice share estimation model in order to predict product demand.

In this paper, we begin by proposing the founding principles of our Usage Coverage Model (UCM): terms, definitions, variable classes and relation classes. Next, we show how it may be integrated into a method of discrete choice analysis to form the complete UCM. Finally, we apply the UCM in a brief case study of cutting a wood board with a jigsaw tool in order to exemplify how the model may be used to predict customer demand. We conclude by reaffirming the utility of the UCM model and outlining the further research work that will be done in the future.

\section{THE USAGE MODEL: BASIC PRINCIPLES}

Our objective is to deliberately put the emphasis on the fitting of a set of product usages with a given product. We call this fitting or adequacy the usage coverage of a product solution. Our primary objective is to define a representation language of customer usage. Further, we want to determine a customer choice model based on usages and an inference mechanism for determining the usage coverage of a product regarding a set of expected usages.

In our usage model, a product, defined by its design parameter vector $X$, must be adapted at best to a set of customer usages $U$ (see figure 2).

\section{$X$ are product design parameters.}

Let us use the example of the expected usage "to cut wood sticks and boards of defined materials and dimensions" throughout this paper. This example may correspond to the market situation described above. Indeed, this is a technical domain for which the definition of the expected usages is very important and the subjective (sensorial, aesthetic, perceptual...) expectations are minor for an experienced handyman or craftsman. In addition, a number of saw categories may deliver an expected cutting service with different effectiveness, efficiency and comfort results. Let us mention the main categories (see table 1): jigsaws, bow saws, panel saws, knife saws, circular saws, miter saws, chain saws, band saws. These tool categories are more or less adapted to a given cutting usage. Moreover, when considering a set of cutting usages (situations), one tool of a given category might cover more or less successfully the entire set of expected usages. For instance, a conventional jigsaw can primarily cover the whole set of expected cutting usages providing the thickness dimension is 
lower than $4.5 \mathrm{~cm}$ - i.e. it relates to the traditional length of cutting blades for jigsaws -. In other usage situations - beyond $4.5 \mathrm{~cm}$ of wood thickness -, a jigsaw is inadequate; only a band saw may exceed this dimension but, as a consequence, it is not well adapted to any type of wood. Imposing the use of a unique saw results in a partial coverage of cutting usages.

Table 1: The main wood saw categories

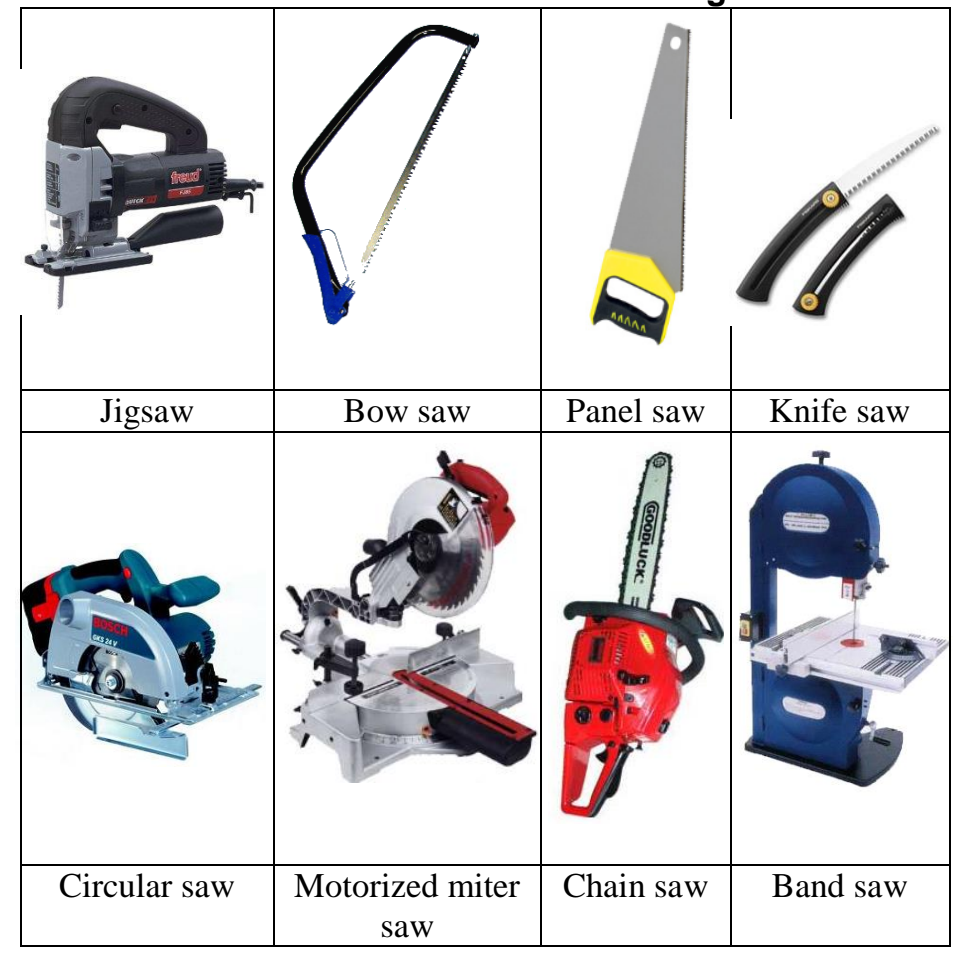

In general, the results of product usage by a customer may depend upon his/her degree of skill, let us say the $C_{s}$ vector. For instance, a novice handyman may fail to cut a thick wood board of hard wood. This notion is rarely taken into account when building customer demand models.

\section{$C_{s} s$ are user-related parameters that affect performances.}

Moreover, the definition of the acceptability threshold of a performance is also customer dependent. For instance, a demanding customer may reject a board cut with insufficient accuracy or a peeling or jagged cut. Every customer has his/her own opinion on acceptable performance thresholds or bounds that he/she is willing to tolerate. Let us call $C_{b}$ these acceptable bounds.

$C_{b}$ are user acceptable bounding constraints of service performances.

Finally, within these acceptable bounds, the obtained performances are considered acceptable and a usage can be completed. But, the customer may express some preferences about the degree of service quality perceived. Let us call $C_{p}$ the customer preferences.

$$
C_{p} \text { are user preferences of performance. }
$$
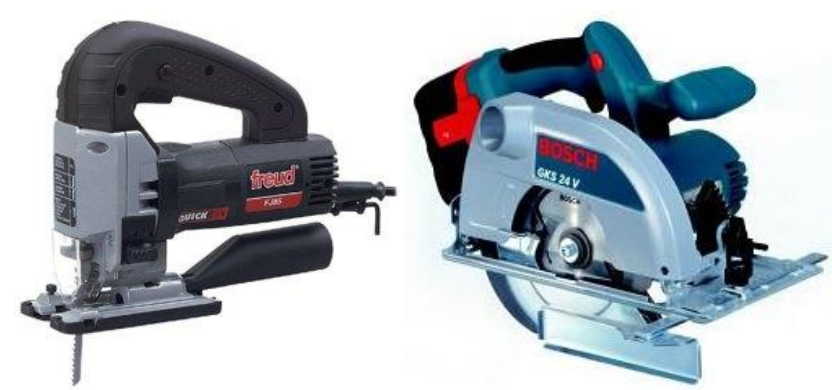

which Design $X$...

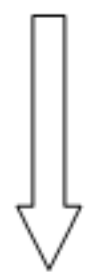

knowing I am a customer with accepted bounds $\boldsymbol{C}_{\boldsymbol{b}}$ for service performances, some skills $C_{s}$ and some preferences $C_{p}$

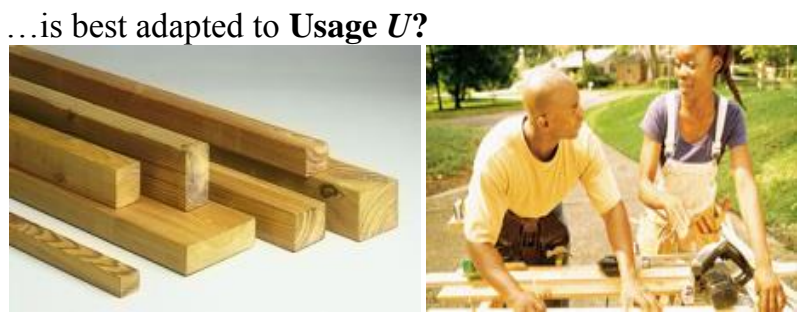

Figure 2: Objective of a design product : to be
adapted at best to a given set of usages $U$

We determine that the performances of the service provided by a given product $X$ handled by a user with skill $C_{s}$ are acceptable when performances respect the bound $C_{p}$ and, in that case that the perceived service quality depends on some preferences $C_{p}$. Finally, let us call $S$ the user-related parameters that influence acceptable performance bounds and preferences and, consequently, the design choice. It should be noted that there is not a strict partition between $C_{S}$ and $S$. For example, gender belongs to $C_{S}$ and $S$ vectors since it may influence the performance achieved ( $C_{s}$ vector) and it may also influence how products are viewed in a choice situation ( $S$ vector).

$S$ are customer demographics which influence choice behavior.

In the following, we prefer to consider a product as a service provider to realize some expected usage. This choice has several strong advantages. First, it allows us to consider not only a physical product but a mix of products-services; second, the usage context surrounding products is made more apparent 
when viewed in this way. Let us propose a series of structuring definitions, starting by the one of a service

A service consists in transforming an initial state of the customer environment into a final state, the discrepancies between the initial and the final stages being mostly desirable.

The initial state is the part of the environment that will be transformed by the product use (e.g., the board to cut). The final state is what has been changed (e.g., the board cut into two parts and, unfortunately, some sawdust). Let us note that what has been changed is not necessarily desired like sawdust. This representation of a service as a transformation (see figure 3) allows a clear definition of service performances and a useful distinction between these performances.

Service performances $Y$ are of two kinds: they feature the results of the transformation by the service and they feature the transformation conditions.

Let us call $Y_{r}$ the first kind of transformation results characteristics and $Y_{t}$ performances characteristics of the transformation conditions. Then, the entire performance vector is:

$$
Y=\left(Y_{r}, Y_{t}\right)
$$

The $Y_{r}$ vector represents the measures of the end performances of the resulting service.

Examples of $Y_{r}$ for the "cutting wood" problem include the precision or the planarity. The performances related to the transformation conditions must not refer to any resulting design characteristics but rather to the manner in which the transformation (design use completion) occurs.

The $Y_{t}$ vector represents the measures of the performances related to the way the service is delivered.

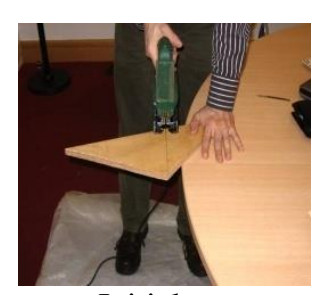

Initial state (what will be transformed)

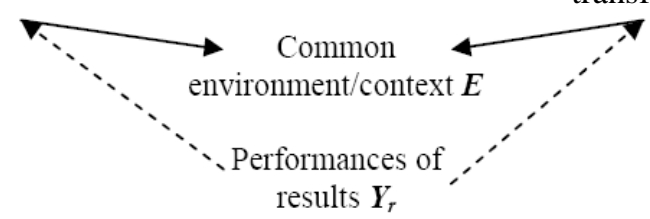

Figure 3: The customer is seen as a product user and a product is seen as a service provider
Examples of $Y_{t}$ include the linear_speed or the noise. Transformation conditions may be enumerated after the TEMIF acronym - Time, Energy, Material, Information, Flows -. Flows correspond to any other type of flow (forces or perceptions like safety, comfort, noise...). They may be considered as performances if a customer has related specifications or preferences.

The so-called transformation occurs in a service environmental context $E$ which is common to both initial and final states and influences the service performances. More generally, it includes the description of the conditions of an elementary service to deliver. For instance, an environmental context of the "to cut a wood board" service may be the wood type, the cut instruction (e.g. length, thickness), the presence of a workbench, the location of the cutting operation, etc.

Variable set $E$ represents any variables that describe the conditions under which the product is used to provide the service.

\section{THE USAGE MODEL: GENERAL MODEL}

In the present usage coverage model (see figure 5 for a global picture), $Y_{r}$ and $Y_{t}$ performances depend both on the design solution considered $X$, the usage context $E$ and the customer skills $C_{s}$. It is expressed by:

$$
\left\{\begin{array}{l}
Y_{r}=f_{r}\left(X, E, C_{s}\right) \\
Y_{t}=f_{t}\left(X, E, C_{s}\right)
\end{array}\right.
$$

Functions $f_{r}$ and $f_{t}$ are called performance equations since they, most of the time, define explicitly the service performances from physics models or empirical studies (virtual or real design of experiments that lead to performance metamodels, see [15]). The elicitation of theses equations is of the utmost importance as soon as we want to study the mapping between given usages and a given product design $X$ (see next chapters).

The performance bounds $C_{b}$ and the performance preferences $C_{p}$ are dependent on user-related parameters $S$.

It is now time to define what a usage is. We first provide the definition of a usage need. Then, we propose three definitions of effective usages given a product design $X$ : feasible usage, acceptable usage and preferred usage, ranging from the less to the most constraining definition of a usage.

A usage needed is a set of expected usage contexts $E_{i}$ associated with a usage frequency $F_{i}$.

This usage frequency $F_{i}$ is the number of times the product is yearly used for the given usage context $E_{i}$. A usage needed is then expressed by:

$$
U_{\text {needed }}=\left\{\left(E_{i}, F_{i}\right)\right\}
$$


An example of a usage needed set is provided in table 2, where a given customer expects to successfully cut $F_{1}$ wood sticks and $F_{2}$ wood boards per year. It is interesting to note that, in practice, the usage contexts are themselves value sets since they are defined as Cartesian products of domains.

Table 2: Example of a usage needed set composed of two usage contexts of different usage percents

\begin{tabular}{|c|c|c|}
\hline $\begin{array}{l}\text { Definition of } \\
\text { usage needed }\end{array}$ & Usage contexts & $\begin{array}{c}\text { Usage } \\
\text { frequency }\end{array}$ \\
\hline & $E_{1}=\left\{\begin{array}{c}\text { material }=\{\text { wood }\}, \\
\text { thickness } \in[5,40], \\
\text { length } \in\{5,60\}, \\
\text { workbench }=\{\text { no }\}\end{array}\right\}$ & $F_{1}$ \\
\hline & $E_{2}=\left\{\begin{array}{l}\text { material }=\{\text { wood }\}, \\
\text { thickness } \in[10,30], \\
\text { length } \in\{20,1000\}, \\
\text { workbench }=\{\text { yes }\}\end{array}\right\}$ & $F_{2}$ \\
\hline
\end{tabular}

A feasible usage is the subset of needed usage contexts that can be fulfilled by a given design $X$.

It means that we look at accomplishing the minimal service so as to be feasible, i.e. effectively cutting a wood board or stick defined by $E_{i}$ without any user requirement neither on the quality of the resulting performances $Y_{r}-$ e.g. the precision or the surface_rugosity -, nor on the preferences on the service processing $Y_{t}$ - e.g., the linear_speed or the noise -. It can be expressed by:

$$
\begin{gathered}
U_{\text {feasible }}\left(X, U_{\text {needed }}, C_{s}\right)= \\
\left\{\begin{array}{c}
\left(E_{i}^{*}, F_{i}\right), \quad \text { such that } \\
\left(E_{i}, F_{i}\right) \in U_{\text {needed }} \\
\text { and } E_{i}^{*} \subseteq E_{i} \\
\text { and } Y_{r_{i}}=f_{r}\left(X, E_{i}^{*}, C_{s}\right) \text { is feasible }
\end{array}\right\}
\end{gathered}
$$

A "feasible performance" in the aforementioned formula means for instance that cutting the board is possible whatever the linear speed and the cutting quality. For cutting the board, the linear speed must be strictly positive, which requires a minimal value of horizontal force and may be impossible by the user ability $\left(C_{s}\right.$ vector).

Figure 4 graphically represents the calculation of the feasible usage $U_{\text {feasible }}$ from the needed usage $U_{\text {needed }}$. For each needed usage context $E_{i}$ it amounts to shrink its domain values to its feasible part $E_{i}^{*}$; this domain reduction being expressed by $E_{i}^{*} \subseteq E_{i}$. As soon as one domain of $E_{i}^{*}$ becomes empty, the initial usage context $E_{i}$ cannot be fulfilled, even partially, i.e., with certain value ranges.

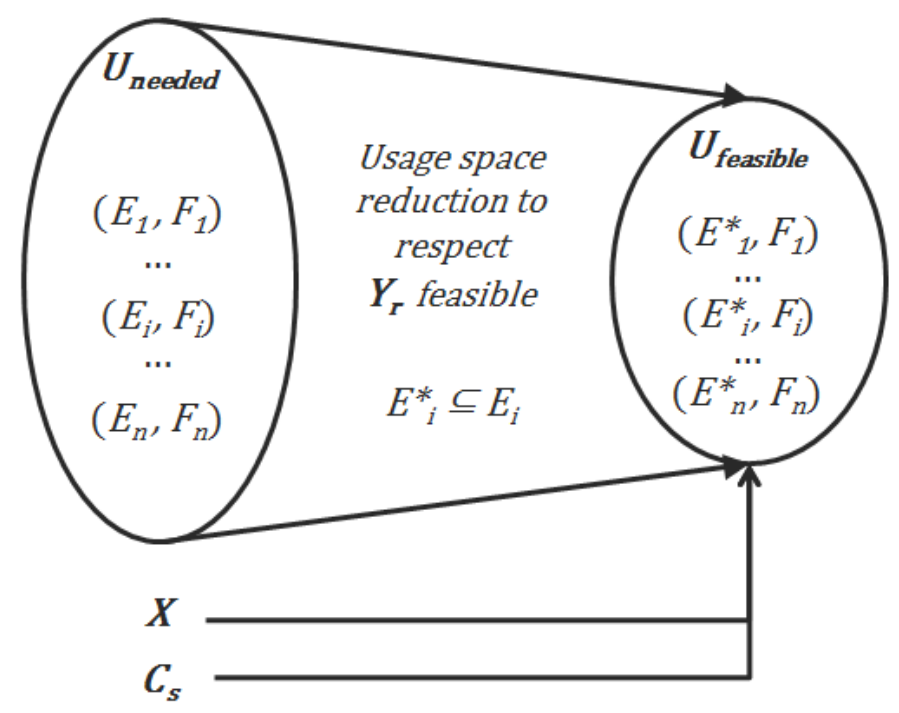

\section{Figure 4: Calculation of the feasible usage set $U_{\text {feasible }}$ from the needed usage set $U_{\text {needed }}$}

An acceptable usage is the subset of a usage need that is fulfilled by a given design $X$ and which is compliant with the required quality level of the service results.

It can be expressed by:

$$
\begin{gathered}
U_{\text {acceptable }}\left(X, U_{\text {feasible }}, C_{s}, C_{b}\right)= \\
\left\{\begin{array}{c}
\left(E_{i}^{*}, F_{i}\right), \quad \text { such that } \\
\left(E_{i}, F_{i}\right) \in U_{\text {feasible }}\left(X, U_{\text {needed }}, C_{s}\right) \\
\text { and } E_{i}^{*} \subseteq E_{i} \\
\text { and } C_{b}\left(Y_{r_{i}}\right) \text { with } Y_{r_{i}}=f_{r}\left(X, E_{i}^{*}, C_{s}\right)
\end{array}\right\}
\end{gathered}
$$

The expression $C_{b}\left(Y_{r}\right)$ meaning that the bounding constraints $C_{b}$ on performances $Y_{r}$ are respected. For instance, a cutting service may be acceptable if and only if the precision and the planarity of the cutting surface area is sufficient. Otherwise, the customer would reject the service and throw or recycle the processed wood parts.

A preferred usage is the possible subset of a usage need that is fulfilled by a given design $X$ and which is compliant with the required quality level of the service results as well as with the maximum level of comfort, safety and pleasure during the service processing.

It can be expressed by:

$$
\left\{\begin{array}{c}
U_{\text {preferred }}\left(X, U_{\text {acceptable }}, C_{s}, C_{b}, C_{p}\right)= \\
\left(E_{i}^{*}, F_{i}\right), \text { such that } \\
\left(E_{i}, F_{i}\right) \in U_{\text {acceptable }}\left(X, U_{\text {feasible }}, C_{s}, C_{b}\right) \\
\text { and } E_{i}^{*} \subseteq E_{i} \\
\text { and } C_{p}\left(Y_{t_{i}}\right) \\
\text { with } Y_{t_{i}}=f_{t}\left(X, E_{i}^{*}, C_{s}\right)
\end{array}\right\}
$$


For instance, a cutting service is preferred if and only if the precision and the planarity of the cutting surface area is sufficient and the comfort and safety conditions are satisfactory.

Figure 5 may summarize the principles of our usage model.

The major usefulness of such a Usage Coverage Model (UCM) is to be able to choose among several parameterized designs and to optimize it, starting from a targeted set of usage needed. On an analysis point of view, we want to get a graphical image of the need coverage of a given product or a given product family, in comparison with other competing products or product families. We also want to be able to take the different psychological and behavioral customer profiles into account in terms of quality requirements concerning both the service performance results $Y_{r}$ and the service processing conditions $Y_{t}$. From the adopted definitions (eq. 4-6) and the set-based modeling of the usages, it follows that:

$$
\begin{aligned}
U_{\text {preferred }}\left(X, U_{\text {acceptable }}, C_{s}, C_{b}, C_{p}\right) & \\
& \subseteq U_{\text {acceptable }}\left(X, U_{\text {feasible }}, C_{s}, C_{b}\right) \\
& \subseteq U_{\text {feasible }}\left(X, U_{\text {needed }}, C_{s}\right) \subseteq U_{\text {needed }}
\end{aligned}
$$

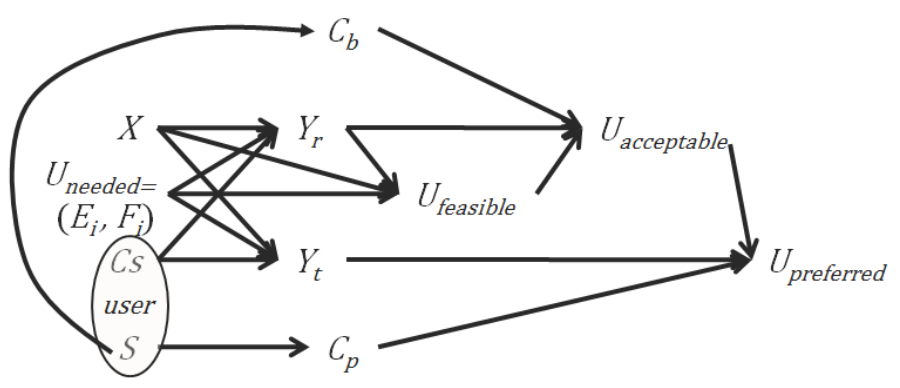

Figure 5: Principles of the usage coverage model

Table 3 summarizes the different usage sets we might get when using the usage coverage model. We might be able to figure out the shrinking ratio of the usage needed set when the service must be fulfilled by a given product-service design $X$ : $U_{\text {feasible }}\left(X, U_{\text {needed }}, C_{s}\right.$ ) (see row \#3 of table 3 ). As soon as the customer requires that the service results be of a certain quality level, the effective usage set acceptable for a given design $X$ is further shrunk into $U_{\text {acceptable }}\left(X, U_{\text {feasible }}, C_{s}, C_{b}\right)$ (see row \#4 of table 3). If, in addition, the customer wants to get these results with a quality of the service processing, this $U_{\text {preferred }}\left(X, U_{\text {acceptable }}, C_{s}, C_{b}, C_{p}\right)$ usage set may be considered as a mathematical fields of preferences.

Our ultimate goal is to develop usage-oriented choice models for narrowing down the choice alternatives for each person to only those which meet his/her requirements. Or, from a product point of view, to assess the competitive advantages of a given product among a set of preexisting competing products defined by slightly different design parameters.
Table 3: Graphical representations of the 4 different usage sets

\begin{tabular}{|c|c|}
\hline Usage type & $\begin{array}{c}\text { Graphical } \\
\text { representation of the } \\
\text { usage set }\end{array}$ \\
\hline$U_{\text {needed }}$ & \\
\hline $\begin{array}{l}U_{\text {feasible }} \\
\left(X, U_{\text {needed }}, C_{s}\right)\end{array}$ \\
\hline$U_{\text {acceptable }}$ \\
$\left(X, U_{\text {feasible }}, C_{s}, C_{b}\right)$
\end{tabular}

Table 4: Basic comparisons of two preferred usage sets for two given design products

\begin{tabular}{|c|c|}
\hline $\begin{array}{c}\text { Comparison of } \\
\text { usage sets }\end{array}$ & $\begin{array}{c}\text { Graphical representation } \\
\text { of the usage sets }\end{array}$ \\
\hline $\begin{array}{l}U_{\text {preferred }}\left(X_{1}\right) \\
\cup U_{\text {preferred }}\left(X_{2}\right)\end{array}$ \\
\\
$U_{\text {preferred }}\left(X_{1}\right)$ \\
$\cap U_{\text {preferred }}\left(X_{2}\right)$
\end{tabular}

For instance, our model might be able to answer questions as (see also table 4): 
- What is the part of the usage set which is best preferred by our own product (it may be empty for a low-cost tool)?

- What is the total surface area of the feasible space covered by our design solution, in comparison with the competing ones?

- For a given customer segment having a certain quality requirement on the service results as well as on the service processing, is our product-service competing (best preferred or at least acceptable) in considering a characteristic usage need scope?

- $\quad$ And so on.

\section{DISCRETE CHOICE ANALYSIS FOR BUILDING A USAGE DEMAND MODEL}

Discrete Choice Analysis (DCA) [16] is used to model product demand by capturing individual customers' choice behavior, in which performance of a given product is considered versus that of competitive products. DCA is based upon the assumption that individuals seek to maximize their personal customer choice utility, $u$, when selecting a product from a choice set. The concept of choice utility is derived by assuming that the individual's $(n)$ true choice utility, $u$, for a design alternative, $i$, consists of an observed part $W$, and an unobserved random disturbance $\varepsilon$ (unobserved utility):

$$
u_{\text {in }}=W_{\text {in }}+\varepsilon_{\text {in }}
$$

While there are a number of DCA techniques popular in literature (e.g., Multinomial Logit, Nested Logit, Mixed Logit), they are distinguished from each other by the degree of sophistication with which they model the unobserved customer choice utility error $\varepsilon$ and heterogeneity in customer preferences. While the Multinomial Logit (MNL) model displays the Independence of Irrelevant Alternatives (IIA) property, ?it is used in this work to demonstrate the proposed design process. In the Multinomial Logit (MNL) model, the coefficients $\beta$ of the observed customer choice utility function $W$ for the product attributes are identical across all customers. However, heterogeneity is modeled by considering demographic attributes $S$ (e.g., customer's age, income, etc.) in the customer choice utility function. Assuming this utility function can be expressed as a linear combination of attributes, $W$ is a function of the product performance $Y_{i n}$ (no distinction made here between $Y_{r}$ and $Y_{t}$ ) and customer demographic attributes $S_{n}$ :

$$
W_{\text {in }}=\beta_{1} Y_{\text {in }}+\beta_{2} S_{n}+\beta_{3}\left(Y_{\text {in }} S_{n}\right)
$$

The product performance $Y_{i n}$, varies for each alternative $i$ based upon the design of the product $X_{i}$, and for each person $n$ based upon his/her specific usage environment $E_{n}$ (i.e. how the product is used) and skill level $C_{s_{n}}$, i.e. product performance is a function of both the product design and how it will be used. Therefore $Y_{\text {in }}$ can be expressed as:

$$
Y_{\text {in }}=f\left(X_{i}, E_{n}, C_{s_{n}}\right)
$$

This functional relationship is established using physics based engineering analysis models or models fit to empirical data (i.e. regression modeling). Thus, choice modeling considering usage context can be viewed as a 2-level hierarchical choice model [15], in which design, usage environment, and skill level are mapped to performance at the lower level, and performance and demographic attributes are mapped to choice utility at the upper level.

The formulation given by Eqs. (9) and (10) implicitly assumes the product is used for a single usage environment, $E_{n}$, for each customer. However, customers may use a single product in multiple usage environments and with multiple usage frequencies, what we gather into a usage needed. For example a saw may be used by a customer both for precision cutting and general construction needs. Therefore, a complementary utility formulation to Eq. (9) can be developed for the case of multiple customer usages, $E_{k}$, where $k$ is the number of unique usage environments for the product. Utility $W_{\text {in }}$ is given by:

$$
W_{\text {in }}=\beta_{1} Y_{\text {in }}+\beta_{2} S_{n}+\beta_{3}\left(Y_{\text {in }} S_{n}\right)+\underbrace{\beta_{4}\left(F_{n, E_{k}} Y_{\text {in, } E_{k}}\right)}_{\text {Multiple Usages }}
$$

In this expression, the $Y_{i n, E_{k}}$ are those performances which are a function of $E_{k}$, whereas $Y_{i n}$ are performance that are independent of specific $E_{k}$. The $F_{n, E_{k}}$ term is the percentage of usage time associated with each specific usage environment $E_{k}$. The $F_{n, E_{k}}$ acts as a weighting factor to place greater weight on the most frequent usages of the product, and less weight on infrequent usages in the utility function.

Estimation of the customer choice utility function $W$ allows the choice share $C S$, for a choice alternative $i$ to be determined by summing over the market population $N$, all probabilities $\operatorname{Pr}_{n}(i)$, of a sampled individual $n$, choosing alternative $i$ from a set of $J$ competitive choice alternatives:

$$
C S(i)=\sum_{n}^{N} P r_{n}(i)=\sum_{n}^{N} \frac{e^{W_{i n}}}{\sum_{k=1}^{J} e^{W_{k n}}}
$$

The set of choice alternatives $J$ may include both the new designed product and the existing competitive alternatives available on the market. The choice alternative set is composed of either actual consumer purchase choices from a set of product alternatives or simulated product choices, such as those resulting from a market survey. Demand for a given design alternative $i$, at time $t, Q(i)_{t}$, is the product of choice share $C S(i)$ by the total market size (or aggregate market segment demand) $D(t)$, for a given product (e.g. circular saw or jigsaw):

$$
Q(i)_{t}=C S(i) D(t)
$$

One challenge in design for usage context, seen in Eq. (10), is that it can be challenging to develop a single product $X_{i}$ which achieves high performance $Y_{i n}$ across many usage environments $E_{n}$, and customer skill levels $C_{s_{n}}$. In product design, a trade-off exists between making several unique 
products or a family of products for usages that are similar, or to make fewer, highly flexible products which cover several usage contexts. To enable this decision-making process, similar usage contexts must first be understood. In our framework, usage environment is generally defined by several variables, for example usage context includes material type, size, hardness and attributes of location for the saw design problem. Additionally, customer skill level may be characterized by one or more attributes. Usage similarity must take into account all usage environments and skill level attributes. Two methods are used for identifying similar usages: Latent Class Analysis (LCA) [17], used when the usage attributes are categorical (e.g., type of wood) or ordinal (e.g. skill level on a scale of 1-3) measures, and Cluster Analysis (CA) [18] when the usage variables are continuous (e.g. thickness of cut to be made). These methods are capable of identifying the optimal number of latent classes or clusters, and all usage contexts which belong to each latent class or cluster. A product development team can use Eq. (13) to estimate the potential choice share for a product design $X_{i}$ for each of the usage context clusters or latent classes for product design decision-making.

\section{APPLYING THE USAGE MODEL FOR "CUTTING WOOD STICKS AND BOARDS"}

In this section we will provide an example set of usage environments and performances, as well as an example of the propagation of usage-specific performance to choice utility. Let us imagine what variable sets may be for the service of "cutting wood sticks and boards" for a given user and with the aid of a given saw tool.

The usage context variables $E$ may be:

- The location where the cutting operation must or is supposed to take place. We may want a more or less flexibility in the tool mobility. Then, we get the following discrete context variable:

location $\in\{$ outside, room, garage,garden $\}$

- The migterial to be cut:

$$
\begin{aligned}
& \text { Material } \\
& \in\{\text { wood, steel, plaster, concrete, break }\}
\end{aligned}
$$

- The type of cut which is planned:

type_of_cut

$\in\{$ right, inclined,curvilinear, groove $\}$

- The cutting trace width, whatever the type_of-cut: width $\in R$

- The cutting curvilinear length, whatever the type_of_cut:

$$
\text { length } \in R
$$

- The presence of a workbench that stabilizes and maintains a wood board or a wood cut during the cutting operation:

\section{workbench $\in\{y e s, n o\}$}

- The presence or not of an electrical outlet in the neighborhood of the place where the need is effective:

$$
\text { outlet } \in\{y e s, n o\}
$$
may be:

The performances $Y_{r}$ corresponding to the service results

- The planarity of the cut surface area:

$$
\text { planarity } \in R
$$

- The precision of the cut surface area relatively to the target and depending of type_of_cut: precision $\in \mathrm{R}$

- The cut surface area rugosity: surface_rugosity $\in\{$ soft, medium,rough $\}$

- The edge lifting: edge_lifting $\in\{y e s, n o\}$

- $\quad$ The purchasing price: price $\in R$

The performances $Y_{t}$ corresponding to the transformation conditions - TEMIF standing for Time, Energy, Material, Information, Fields - may be:

- (T) linear_speed

( $C_{S}$ dependent $)$

- (T) preparation_time

( $C_{S}$ dependent)

- (E) electrical_energy

( $C_{s}$ dependent $)$

- $(\mathrm{M})$

Dust_ratio $=$ Total_dust /(length $\times$ width $)$

- (I) Visibility

- (F) Safety ( $C_{s}$ dependent $)$

- (F) Noise

- (F) Force\&Vibrations

( $C_{S}$ dependent $)$

- (F) Operating_\&_maintenance_costs $\left(C_{s}\right.$ dependent)

The design vector $X$ may be composed of a set of variables, specific to any design solution. In table 5, two design solutions, able to "cut wood sticks and boards" are described by their major design variables: a rotating saw and a jigsaw. Here, our understanding of design vector $X$ is much closer to the marketing attributes any customer may apprehend whenever he/she looks at purchasing a given tool than to the design parameters of Suh in axiomatic design theory [19]. One can note that the composition of $X$ vectors is not necessarily the same for two different design architectures. In table 5, the jigsaw has an additional $X$ component and the speed variable is rotational for the circular saw and is a translational frequency for the jigsaw. Conversely, the composition of the $Y$ vector is common for all the saw architectures since it gathers performances exclusively linked to the service. 
With expressions for $Y_{r}$ and $Y_{t}$ for the service of cutting wood sticks and boards, the consumer data required for estimating the choice model can be collected. A choice-based conjoint survey can be completed by a sample of consumers from a target market segment, characterized by varying usage environments $E$, skill levels $C_{S}$, and demographic attributes $S$. Each model to be estimated will have a different optimal conjoint design and sample size [16]. In this survey, the respondents are provided with a set of saw designs with differing performance levels $Y$, and select their preferred saw design from the choice alternatives. Completion of the survey by all respondents provides the data necessary for estimation of the choice model. Estimation of the choice model allows prediction of the potential choice share for a given saw design $X_{i}$ for a target market characterized by $\left(E, C_{S}, S\right)$, using Eq. 12 .

\section{Table 5: Representation of two design solutions $X$}

\begin{tabular}{|c|c|}
\hline Circular saw & Jigsaw \\
\hline & \\
\hline Power $=1000 W$ & Power $=500$ W \\
\hline Type_of_Blade $=\{w o o d\}$ & Type_of_Blade \\
& $=\{$ wood,steel $\}$ \\
\hline speed $=1 \mathrm{~m} / \mathrm{s}$ & speed $\in[1,15]$ stroke $/ \mathrm{s}$ \\
\hline $\begin{array}{c}\text { Maximal_thickness } \\
\in[10 m m, 40 m m]\end{array}$ & $\begin{array}{c}\text { Maximal_thickness } \\
\in[3 m m, 45 m m]\end{array}$ \\
\hline Light $=\{y e s\}$ & Light $=\{$ no $\}$ \\
\hline Laser $=\{y e s\}$ & Laser $=\{$ no $\}$ \\
\hline Changeable_Blade $=\{$ no $\}$ & Changeable_Blade $=\{y e s\}$ \\
\hline $\begin{array}{c}\text { Dust_Aspiration } \\
=\{\text { possible_ootion }\}\end{array}$ & \begin{tabular}{c} 
Dust_Aspiration $=\{$ no $\}$ \\
\hline Price
\end{tabular} \\
\hline \multicolumn{2}{|c|}{ Price } \\
\hline
\end{tabular}

Prediction of choice share for a given design enables use of the Decision-based Design framework [13] to make engineering design decisions.

In order to provide further explanation of our method, we will use the brief example of a jigsaw tool being used to cut a wood board. For simplicity, we will only concern ourselves with the comfort of the user's wrist, $P_{\text {comfort }}$ as the sole measure of performance:

$$
P_{\text {comfort }}=1-\left|\frac{M_{w}}{M_{w \text { max }}}\right|, \in[0,1]
$$

where $M_{w \max }$ is the maximum amount of wrist torque that the user can provide (an element of skill), while $M_{w}$ is the torque received in the wrist of the user, calculated as:

$$
M_{w}=F_{a}\left(H_{w}+\frac{T_{c}}{2}\right)+F_{c} L_{w}+F_{f} H_{w}-\left(d+L_{w}\right) F_{r}
$$

and is a function of design and context attributes. $H_{w}$ and $L_{w}$ describe the position of the wrist, while $d$ describes the location of the reaction force on the jigsaw slider, all of which are elements related to the product design. Elements of context include $T_{c}$, which is the thickness of the board being cut, and $F_{f}$ and $F_{t}$, which are both functions of the friction factor between the jigsaw and wood. The remaining variables are intermediate variables, which are simply functions of other design and context variables. $F_{a}$ captures the force of the advancing blade on the wood, while $F_{c}$ describes the force of the cutting blade.

With expression for $Y_{t}$ performance $P_{\text {comfort }}$ defined, its use in choice modeling can be demonstrated. A choice-based conjoint survey can be completed by a sample of consumers from a target market segment, characterized by varying usages $U$, skill levels $C s$, and demographic attributes $S$. In this survey, the respondents are provided with a set of saw designs with differing performance levels of performance, including $P_{\text {comfort }}$, and select their preferred saw design from the choice alternatives. Completion of the survey by all respondents provides the data necessary for estimation of the choice model. Estimation of the choice model allows prediction of the potential choice share for a given saw design $X_{i}$ for a target market characterized by $\left(E, C_{S}, S\right)$. An example of the utility function for the two usages demonstrated in Table 3, considering only the single performance $P_{\text {comfort }}$ and a given user characterized by two $S$, age (Age) and income (Inc), is given by (interactions omitted):

$$
\begin{gathered}
W_{\text {in }}=\beta_{1}\left(F_{1} P_{\text {comfort }, 1}\right)+\beta_{2}\left(F_{2} P_{\text {comfort }, 2}\right)+\beta_{\text {age }} \text { Age } \\
+\beta_{\text {inc }} \text { Inc }
\end{gathered}
$$

where $F_{l}$ and $F_{2}$ are the frequencies of usage for usages 1 and 2, and $P_{\text {comfort }, 1}$ and $P_{\text {comfort }, 2}$ are the comforts associated with usages 1 and 2 . This example demonstrates the propagation of a performance measure calculated using $\left(X, E, C_{s}\right)$ to the choice level utility function. The choice model provides prediction of choice share for a given design, enabling use of the Decision-based Design framework [13] to make engineering design decisions.

\section{CONCLUSION}

The usage coverage model (UCM) we proposed in this paper intends to represent a more thorough marketing model for products and services in which the contextualization of usage is 
fundamental. Our model attempts to accomplish this by embodying a paradigm in which customers are understood as product employers and products as service providers. This method of quantifying individuals' performances during product usage is new; it offers the advantage of linking with user experience to introduce the perceived quality of a product's service, as well as to consider particular service delivery conditions. In this way, the UCM model is able to distinguish between the quality (or performance) of the product's service results and the quality of the product's service delivery process.

In this paper, the principles of the UCM model - Usage Coverage Model -, i.e. terms, variable definitions and classes as well as relation classes are proposed. Four kinds of usage sets are defined. A Discrete Choice Analysis (DCA) model is proposed to build demand models that better correlate design parameters, product-service performances and demographic variables on the one side, and usage contexts, usage frequency ratios, user skills on the other side.

This paper has presented the UCM model through the example of the choice of a saw tool for a cutting usage. Much work is being carried out to further develop the framework. We are currently creating adapted questionnaires to administer to customer panels to investigate cutting usages. The use of Latent Class Analysis (LCA) [17] and/or Cluster Analysis (CA) [18] to identify clusters of similar usages is being investigated. A method to narrow down the choice alternatives for a given consumer, based upon his/her usage and preferences, using constraint programming is under development. The physicsbased models of performances to provide unique performance levels for each usage context of each individual are currently under development. Usage coverage metrics are being developed to quantify the usage coverage of a given design. Extending these usage coverage metrics to product families is also a future goal.

\section{ACKNOWLEDGEMENTS}

The grant support from National Science Foundation (CMMI0700585) to the authors at Northwestern University are greatly appreciated. We are grateful for the supplemental funding from the NSF International Research and Education in Engineering program that supported the summer visit of Wei Chen and Chris Hoyle to Ecole Centrale Paris, France and made this research collaboration possible.

The grant of the Chinese Scholarship Council has allowed to support the work of Jiliang Wang.

\section{REFERENCES}

1. Rossi, P. E., Allenby, G. M., and McCulloch, R., 2005, Bayesian Statistics and Marketing, John Wiley and Sons, Ltd., Hoboken, NJ.

2. Ratneshwar, S., and Shocker, 1991. A. "Substitution in Use and the Role of Usage Context in Product
Category Structures." J. Marketing Research. Vol. XXVIII, 281-95.

3. Christensen, H., S. Cook, and T. Hall, 2005. "Marketing Malpractice: The Cause and the Cure." Havard Business Review.

4. Petiot J.-F., Yannou B., 2004, "Measuring consumer perceptions for a better comprehension, specification and assessment of product semantics", International Journal of Industrial Ergonomics, 33(6), 507-525.

5. Green, M. G., Palani, R. P. K., and Wood, K. L., 2004, "Product Usage Context: Improving Customer Needs Gathering and Design Target Setting," Proceedings of ASME 2004 DETC \& CIE Conference, Sept. 28-Oct. 2, Salt Lake City, UT.

6. Green, M. G., Tan, J., Linsey, J. S., Seepersad, C. C., and Wood, K. L., 2005, "Effects of Product Usage Context on Consumer Product Preferences," Proceedings of ASME 2005 IDETC \& CIE Conference, Sept.24-28, Long Beach, CA.

7. Green, M. G., Linsey, J. S., Seepersad, C. C., and Wood, K. L., 2006, "Frontier Design: A Product Usage Context Method," Proceedings of ASME IDETC \& CIE Conference, Sept. 10-13, Philadelphia, PA.

8. Coatanea E., Alizon F., Christophe F., Yannou B., 2008, "Selecting technology alternatives for product families through technological coverage and functional verification", IDETC: ASME International Design Engineering Technical Conferences \& Computers and Information in Engineering Conferences, August 3-6, New-York, USA.

9. Li, H., and Azarm, S., 2000, "Product Design Selection under Uncertainty and with Competitive Advantage," Transactions of ASME: Journal of Mechanical Design, 122(4), 411-418.

10. Besharati, B., Azarm, S., and Farhang-Mehr, A., 2002, "A Customer-Based Expected Utility Metric for Product Design Selection," Proceedings of ASME 2002 IDETC Conference, September 29-October 2, Montreal, Canada.

11. Michalek, J. J., Feinberg, F. M., and Papalambros, P. Y., 2005, "Linking Marketing and Engineering Product Design Decisions via Analytical Target Cascading," Journal of Product Innovation Management, 22(1), 42-62.

12. Michalek, J. J., Ceryan, O., Papalambros, P. Y., and Koren, Y., 2005, "Manufacturing Investment and Allocation in Product Line Design Decision Making," Proceedings of the 2004 DETC: ASME Design Engineering Technical Conferences, Sept. 26-28, Long Beach, CA.

13. Wassenaar, H. J., and Chen, W., 2003, "An Approach to Decision-Based Design with Discrete Choice Analysis for Demand Modeling," Transactions of the ASME: Journal of Mechanical Design, 125(3), 490497. 
14. Kumar, D., Hoyle, C., Chen, W., Wang, N., GomezLevi, G., and Koppelman, F., 2007, "A Hierarchical Choice Modeling Approach for Incorporating Customer Preferences and Market Trends in Vehicle Package Design," Proceedings of 2007 ASME DETC/CIE Conference, Las Vegas NV.

15. Simpson T.W., Peplinski, J.D., Koch P.N. and Allen, J.K., 2001, "Metamodels for Computer-based Engineering Design: Survey and recommendations", Engineering with Computers, 17, 129-150.

16. Ben-Akiva, M., and Lerman, S. R., 1985, Discrete Choice Analysis, MIT Press, Cambridge, MA.

17. McCutcheon, A. L., 1987, Latent Class Analysis, Sage Publications, Beverly Hills, CA.

18. Johnson, R. A., and Wichern, D. W., 2002, Applied Multivariate Statistical Analysis, Prentice Hall, Upper Saddle River, NJ.

19. Suh N., 1993, The Principles of Design, Oxford University Press, Oxford. 